\title{
INVESTIGATION OF SOME ISSUES OF FULL OPTICAL MULTIMEDIA NETWORKS
}

Deployment of optical communication technologies into the core networks has allowed improvements in the transmission characteristics. Further penetration towards the end-user customers has brought online multimedia services. Increasing demand for a quality of service and new types of multimedia services challenges current state of the art technologies and drives not only coming technological trends. Recently emerging DPSK (Differential Phase Shift Keying) format is described and impact of OSNR (Optical Signal to Noise Ratio) level and laser linewidth on its performance is investigated in the environment of Virtual Photonics simulation software. Behavior of used modulation format within WDM (Wavelength-Division Multiplexing) system is examined at four channel's WDM system.

\section{Introduction}

A progress in information and communication technologies has allowed high bit rates being affordable to mass market. Multimedia services has got infrastructure with sufficient performance and they could reach their target customers. The expansion of multimedia services and decreasing cost for transmitted megabytes are synergic driving forces. The demand for higher transmission capacity and higher efficiency pushes optical communication to create more complex designs and solutions. In this process, a lot of various effects occur. Optimal setting is a trade-off between introduced distortions due to phenomena and their relations in the optical fibre and our capabilities to suppress them. The possibility of pure physical experiments is unacceptable from more points of views. The response is the application of simulation methods as one part of developing and implementation process. The simulation accuracy and depends from simulation models and assumptions.

Current demand for longer distances and higher bit rates per channel are met by a replacement of primary used on-off keying (OOK) by differential phase shift keying (DPSK) due to its higher sensitivity and robustness against nonlinear effects $[1,2,3]$. Theoretical knowledge was summarized by Gnauck et al. [4]. We wish to deeper investigate DPSK behavior, particularly impact of the laser linewidth and nonlinear effects in the presence of more optical signals with different wavelengths and simulate wavelength division multiplexing system. Our work is carried out in simulation environment of VPI Virtual Photonics.

Second chapter briefly introduces a differential phase shift keying (DPSK) format. Third chapter will show performed simulations and their results. Results are summarized and discussed in conclusion.

\section{Differential phase shift keying}

Phase-shift-keyed (PSK) formats carry the information in the optical phase itself. The receiver has to compare detected signal with reference signal and extract information. Due to the lack of an absolute phase reference in direct-detection receivers, the phase of the preceding bit is used as a relative phase reference for demodulation. This results in DPSK formats, which carry the information in optical phase changes between bits. DPSK has got several advantages against ASK (Amplitude Shift Keying) modulation thus it is not surprising that many of the recent long-haul WDM transmission records at per-channel rates of 10 and $40 \mathrm{~Gb} / \mathrm{s}$ are now held by systems based on DPSK.

Optical systems based on DPSK are not new. DPSK was extensively studied in the late 1980s and early 1990s for use mainly in single-span fiber-optic systems employing coherent receivers as well as in the context of free-space optical communications, where the 3-dB sensitivity advantage over OOK could be exploited. When erbium-doped fiber amplifiers (EDFAs) were introduced, interest in coherent systems declined. For about a decade, OOK-based WDM systems using optical-amplifier repeaters dominated the research in long-haul optical communications. Interest in DPSK reemerged several years ago, as WDM systems were pushed to ever-higher levels of performance [4].

In the DPSK format, optical power appears in each bit slot, with the binary data encoded as either a 0 or $\pi$ optical phase shift between adjacent bits. The optical power in each bit can occupy the entire bit slot (NRZ-DPSK) or can appear as an optical pulse (RZ-DPSK). The most obvious benefit of DPSK when compared to $\mathrm{OOK}$ is the $\sim 3-\mathrm{dB}$ lower OSNR required to reach a given BER. This can be understood by comparing the signal constellations for DPSK and OOK, as shown in Fig. 1. For the same

\footnotetext{
* Dusan Sabol ${ }^{1}$, Jozef Dubovan ${ }^{2}$, Miroslav Markovic ${ }^{2}$, Milan Dado ${ }^{2}$

${ }^{1}$ Dept. of Electrical, Electronical and Mechanical Engineering, University College Dublin, Ireland, E-mail: dsabol@centrum.sk

${ }^{2}$ Dept. of Telecommunications and Multimedia, FEE, University of Zilina, Slovakia,
} 


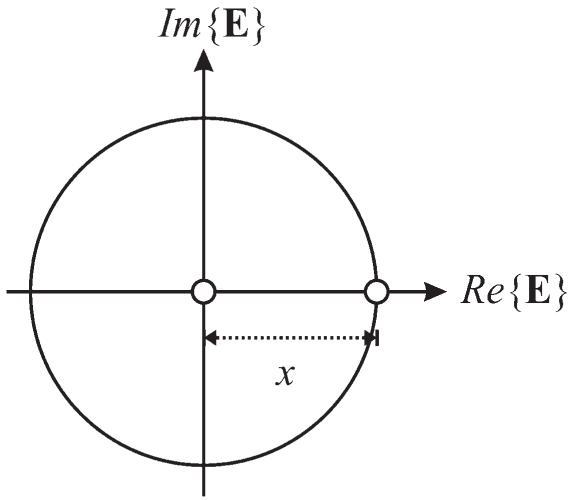

On - Off Keying

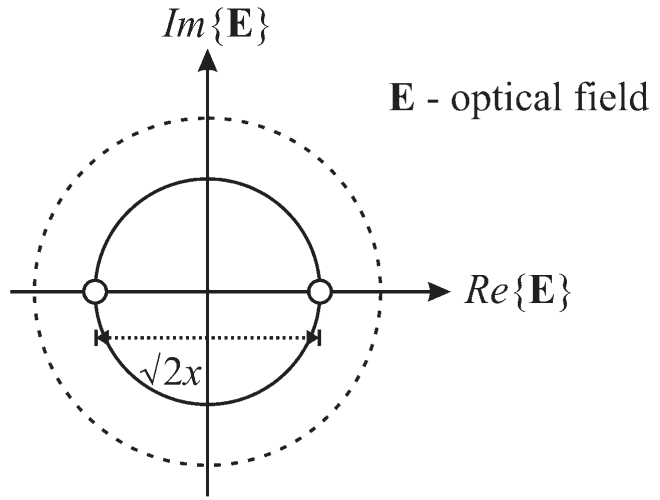

Differential Phase Shift Keying

Fig. 1 Signal constellation of OOK and DPSK modulation [4]

average optical power, the symbol distance in DPSK (expressed in terms of the optical field) is increased by $\sqrt{2}$. Therefore, only half the average optical power should be needed for DPSK as compared to OOK to achieve the same symbol distance. This $\sim 3 \mathrm{~dB}$ benefit of DPSK modulation can be only extracted by using balanced detection [4].

The lower OSNR requirement of DPSK can be used to extend transmission distance, reduce optical power requirements or relax component specifications.

DPSK with balanced detection has been demonstrated to offer large tolerance to signal power fluctuations in the receiver decision circuit because the decision threshold is independent of the input power. DPSK is more robust to narrow-band optical filtering than OOK, especially when balanced detection is employed. Numerical simulations and experiments have shown DPSK to be more resilient than OOK to some nonlinear effects. This results from the fact that: i) the optical power is more evenly distributed than in OOK (power is present in every bit slot for DPSK, which reduces bit-pattern-dependent nonlinear effects) and ii) the optical peak power is $3 \mathrm{~dB}$ lower for DPSK than for OOK for the same average optical power. Finally, an extension to differential quadrature phase-shift keying (DQPSK) and other multilevel formats should enable higher spectral efficiency and greater tolerance to chromatic- and polarization-mode dispersion [5]
In the next section, we come to simulations. In First step we want to reproduce experimentally obtained results achieved by Avlonitis et al [5] that our simulation enviroment will be in agreement with their results. Then we proceed in our simulation, add some WDM network components and examine the impact of the laser linewidth and Kerr's effect on the DPSK signal propagation in 4 channel WDM system.

\section{Simulation}

All simulation work was carried out in the simulation environment of VPI Photonics, which allows various conditions and assumptions. In order to get realistic data, we take a work of Avlonitis et al. [5] and try to get the same results from simulation. They looked on the effect of various values if OSNR and the laser linewidth on the narrowband filtered DQPSK modulation format on BER in the back-to-back configuration. They explore BER as a function of OSNR for different laser linewidth. Their equivalent scheme in VPI Photonics environment is shown in Fig. 2.

NRZ-DQPSK source consists of CW laser followed by two dual stage Mach-Zehnder modulators. The used bit rate was $10 \mathrm{~Gb} / \mathrm{s}$. The source output power and power density of AWGN (Additive White Gaussian Noise) was set on the values that corresponded to OSNR $=5 \mathrm{~dB}$. The attenuator modified level of OSNR in the

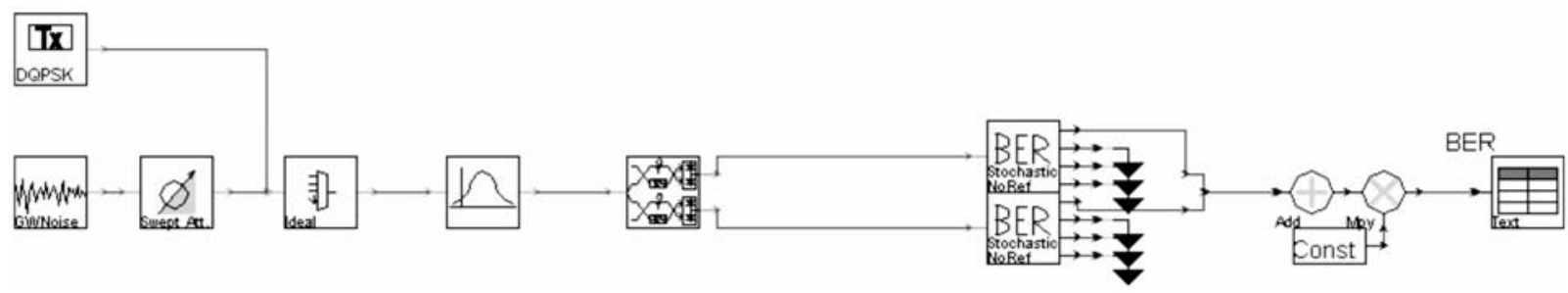

Fig. 2 Simulation scheme of BER $=f(O S N R)$ for DQPSK filtered signal in back-to-back configuration [6] 
BER $=f($ OSNR $)$

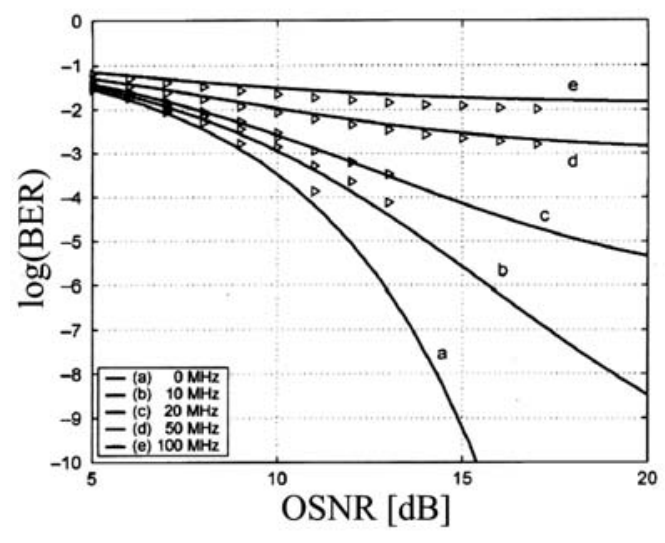

a)

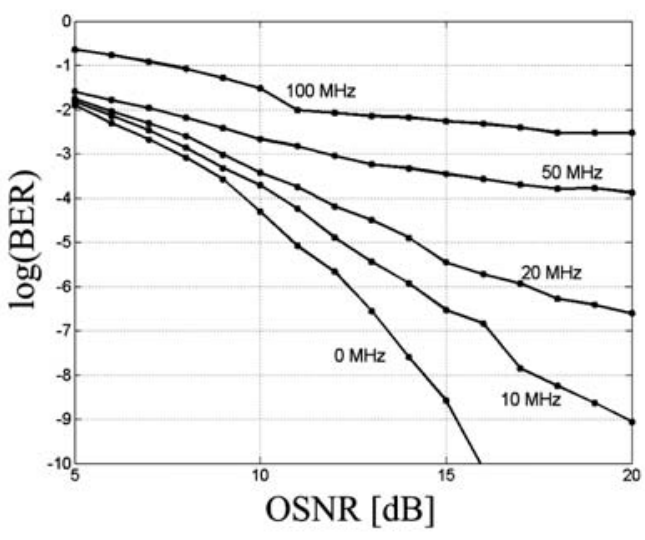

b)

Fig. 3 a) experimental and b) simulation results

range from 5 to $20 \mathrm{~dB}$. The signal passed through 1st order Gaussian bandpass filter with $6 \mathrm{GHz}$ linewidth (spectral efficiency equals to $1.67 \mathrm{~b} / \mathrm{s} / \mathrm{Hz}$ ), which was identical with the experiment. The signal was demodulated in the optical delay line demodulator and detected at the balanced receiver. At the end, BER was assigned to every value of OSNR. It was done for $0,10,20,50$ and $100 \mathrm{MHz}$ laser linewidth. Both experimental and simulation results are plotted in Fig. 3.

Next step involved the increase of the bit rate up to $40 \mathrm{~Gb} / \mathrm{s}$ and filter bandwidth expansion up to the equal value of spectral efficiency. Unfortunately, the simulation provided satisfied results only up to $15 \mathrm{~dB}$ or $\mathrm{BER}=10^{-4}$, which was not sufficient. The
$40 \mathrm{~Gb} / \mathrm{s}$ bit rate variant was not estimated with a desired likelihood and will not be considered in further simulation.

Another simulation investigated DPSK modulation. The setup was modified by replacing DQSK (Differential Quadrature Shift Keying) components for DPSK components. DPSK source consisted of a CW (Continuous-Wave) laser and one dual stage MachZehnder modulator. Laser power was set on one half of the previous value and bandpass filter had a half bandwidth in order to keep the same effect of a filter on the modulated signal. One delay line interferometer with a single balanced receiver was employed and BER (Bit Error Ratio) estimation provided the same module (Fig. 4).
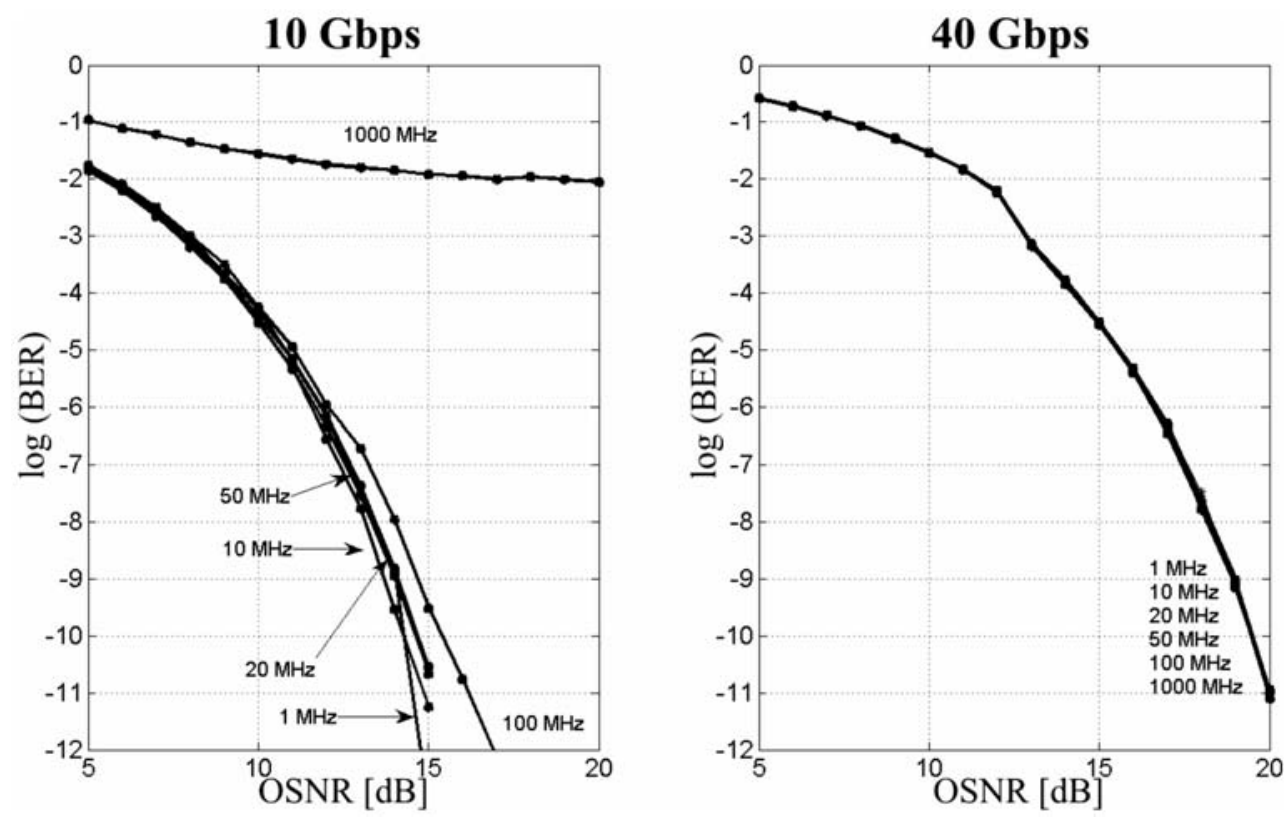

Fig. 4 BER vs OSNR for $10 \mathrm{Gbs}$ and $40 \mathrm{~Gb} / \mathrm{s}$ DPSK format 
The transmission bit rate was $10 \mathrm{~Gb} / \mathrm{s}$. The output data corresponds to theoretical predictions that DPSK is more resistant against phase noise. The divergence of particular curves is not rapid, but a typical flat waveform occurs for $1 \mathrm{GHz}$ linewidth. The OSNR difference between DQPSK and DPSK shows better performance of DPSK. Its benefit increases with lower BER. The DPSK source generated $40 \mathrm{~Gb} / \mathrm{s}$ stream didn't repeat the specific behavior of coherent systems with phase noise. The simulation at $40 \mathrm{~Gb} / \mathrm{s}$ DPSK might not give us physically reasonable results hence $40 \mathrm{~Gb} / \mathrm{s}$ bit rate will not be simulated.

We choose $10 \mathrm{~Gb} / \mathrm{s}$ data stream in NRZ-DPSK format generated by two different lasers with $2 \mathrm{MHz}$ and $500 \mathrm{kHz}$ linewidth and lase them into the optical fibre. We do the same also for NRZOOK format.

\section{OOK and DPSK performance in the fibre}

In this section, the optical link is connected to four DPSK transmitters and we wish to see an effect of fibre (especially nonlinear effects occurring in WDM systems) on the signal. The transmitter and receiver parameters are identical with previous simulations. Channel spacing is set on $50 \mathrm{GHz}$. The optical fibre is arranged in the $4 \mathrm{~km}$ long fibre loop. Dispersion compensating fibre (DCF) is inserted between transmission fibre, which is nonzero dispersion shifted fibre (NZ-DSF) and measurement devices. Demultiplexing provides the identical filter like in the previous simulations. We monitor optical spectrum, eye diagrams of signal's channels, power levels of selected signal channels and two lower, two upper induced bands [6] (simulation scheme is depicted in the Fig. 5)
OOK format is not sensitive to the used laser linewidth. On other hand, DPSK format performance is dependent on the linewidth of used laser and narrowed linewidth shows better performance. Our comparison shows a case of $500 \mathrm{kHz}$ laser linewidth.

The eye diagrams of signal before the entering of fibre scan are shown in Fig. 6. We notice a large eye opening in both cases. OOK and DPSK format might be distinguished by a time jitter. If we increase a laser linewidth, OOK will have negligible distortion but borders defining an area of DPSK eye diagram (red lines in Fig. 6) will get broader and therefore caused the closing of eye.

We sent the laser output through fibre and wanted to monitor a signal distortion span-by-span, which gives us a $6 \mathrm{~km}$ interval. Unfortunately, VPI Photonics was not able to calculate BER and show proper results of second and higher fibre spans.

Our evaluation is based on the eye diagram comparison. OOK format eye diagrams of all 4 channels are shown in Fig. 7 (left) and the same graphs for DPSK format are in Fig. 7 (right). We might notice a different character of distortion, where OOK format has a lot of amplitude noise in the high level. A noise power after $4 \mathrm{~km}$ transmission through fibre is few orders higher opposite the back-to-back signal. DPSK format after $4 \mathrm{~km}$ fibre propagation is almost unaltered. Both eye diagrams have approximately the same value of opening after the $4 \mathrm{~km}$ fibre span. If we return back to back-to-back setup, Fig. 6, we will see that OOK format has got advantage of $\sim 2.5 \mathrm{~mW}$ higher value $(\sim 8 \mathrm{~mW}$ for OOK against $5.5 \mathrm{~mW}$ for DPSK) compared to DPSK format.

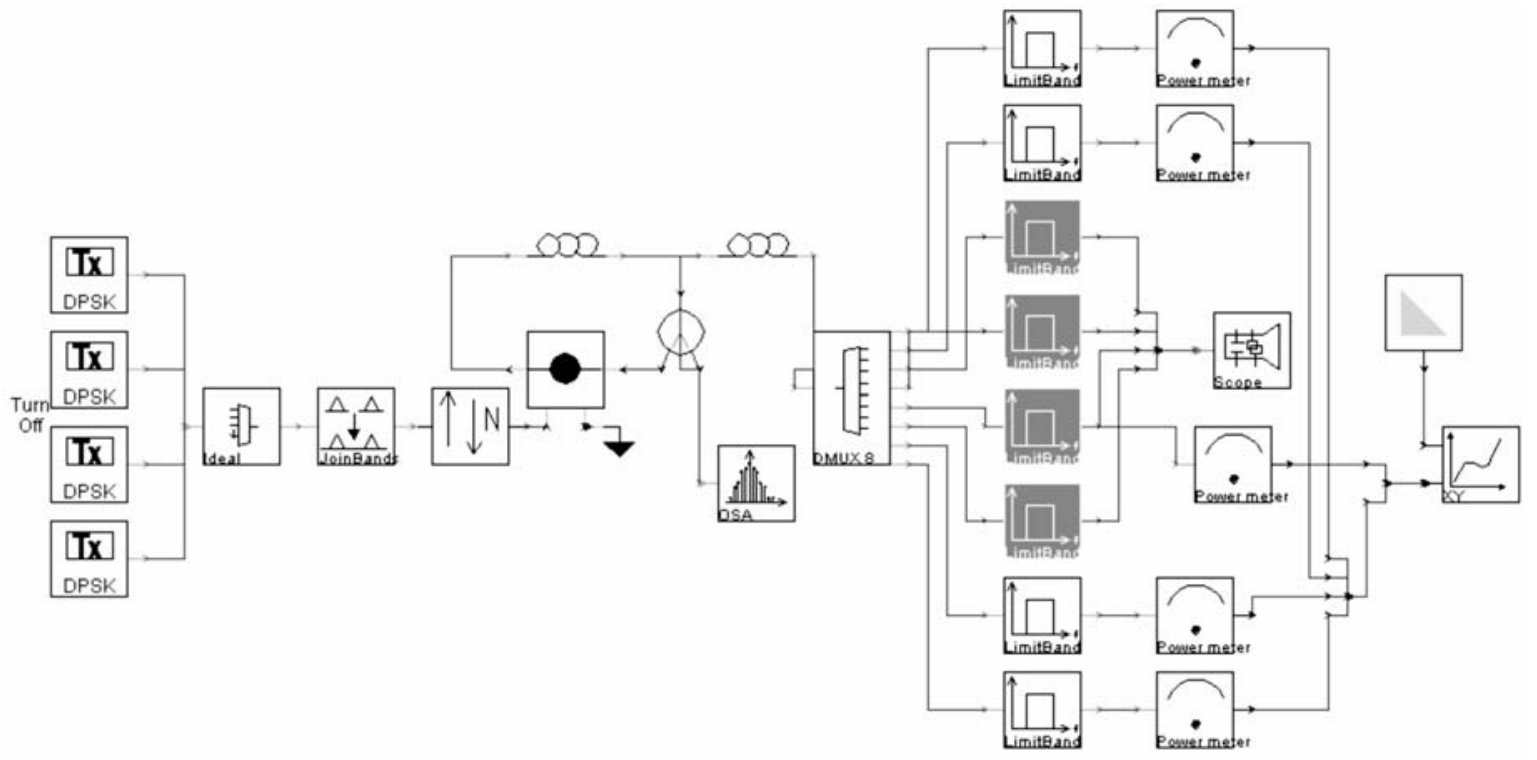

Fig. 5 Simulation scheme of FWM generation 


\section{Eye diagram}
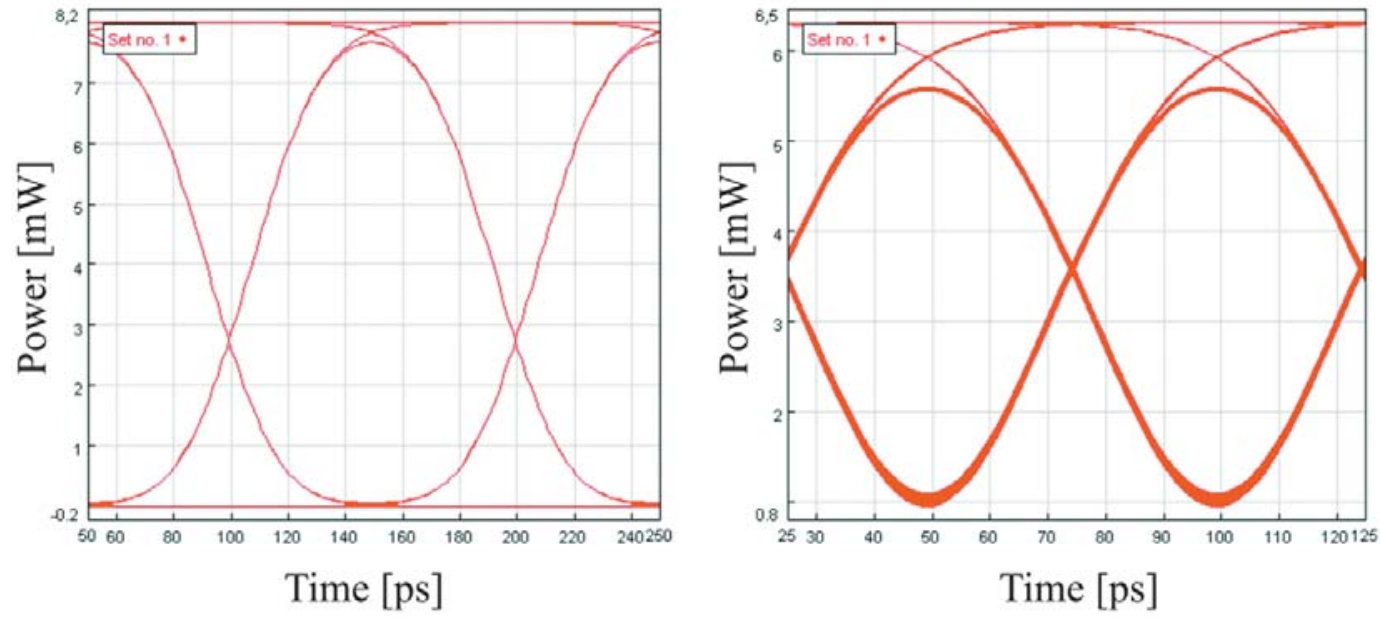

Fig. 6 Eye diagrams of OOK (left) and DPSK (right) transmitters. Laser linewidth $500 \mathrm{kHz}$
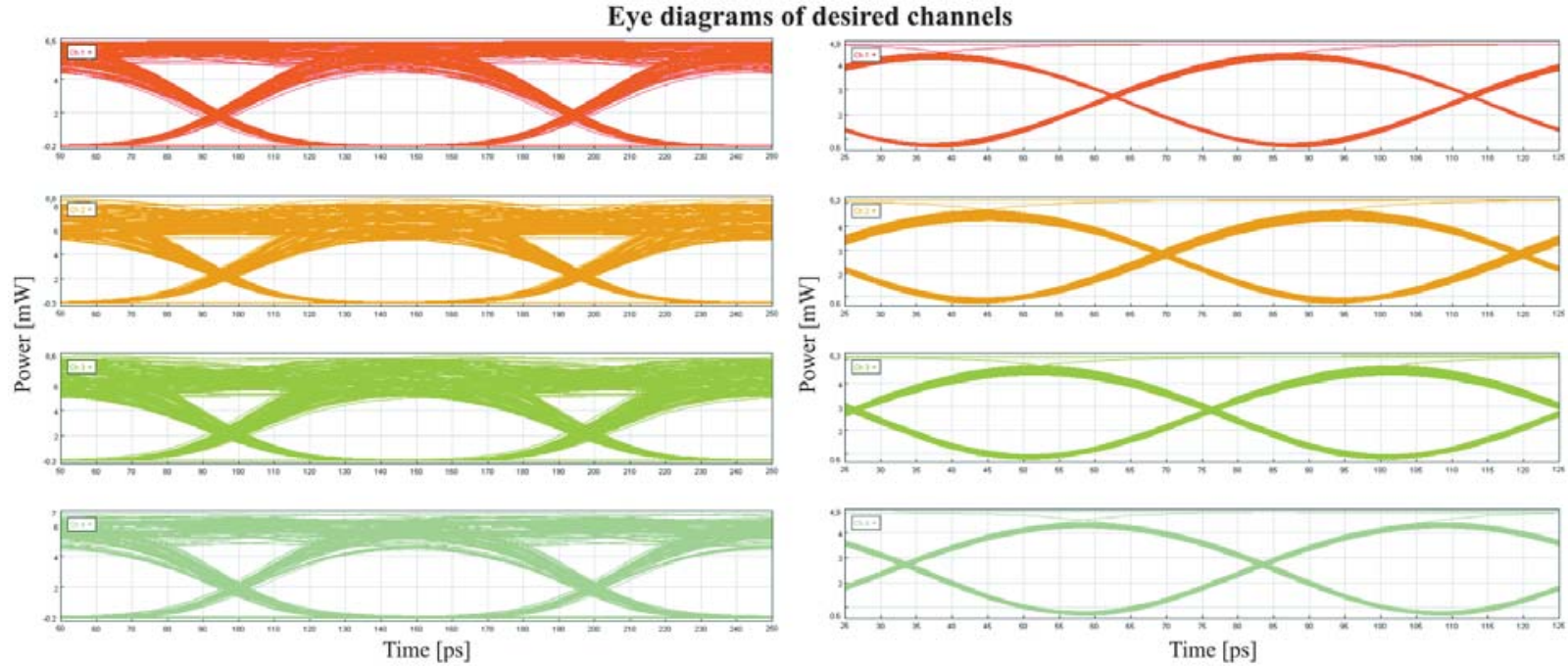

Fig. 7 Eye diagrams after $6 \mathrm{~km}$ span, OOK format(left), and eye diagrams after $6 \mathrm{~km}$ span, DPSK format (right)

Fig. 7 Eye diagrams after $6 \mathrm{~km}$ span, OOK format(left), and eye diagrams after $6 \mathrm{~km}$ span, DPSK format (right)

An excess power of $2.5 \mathrm{~mW}$ has been converted to the noise, which is distributed around the $5 \mathrm{~mW}$ value. It might suggest that DPSK is more resilient against nonlinear processes than OOK. Nonlinear effects are significant at high powers, which means, in the beginning of spans or after amplifiers. Another advantage of DPSK format is in using a balanced receiver which increases sensitivity by $3 \mathrm{~dB}$ and hence might improve BER. But we could not make such an analysis.

\section{Conclusion}

Performance of DPSK and DQPSK transmitters was examined for various values of OSNR and laser linewidth for today's 10 $\mathrm{Gb} / \mathrm{s}$ and future $40 \mathrm{~Gb} / \mathrm{s}$ gold standard bit rates. Model deployed higher bit rate is not absolutely relevant, because it predicts unrealistic behavior for OSNR $>15 \mathrm{~dB}$ or BER $>10^{-4}$. The model of lower bit rate estimation operated reliably and was used for the evaluation of nonlinear effects on the DPSK and OOK modulation formats for two types of optical fibre and two laser linewidths. The simulation shows a significant noise generation in OOK 
signal, which was not the case in DPSK signal. It might sugges higher DPSK resilient against nonlinear effects. DPSK showed a lower distortion and a criterion of BER could not be applied due to computation difficulties. In BER performance DPSK would also have a benefit of balanced detection and might show even better performance.

\section{Acknowledgements}

The reseach presented in this paper was done with the support of COST 291 action.

APVV project COST-0041-06 and APVV project "Technologies for optical signal processing for the next generation optical digital networks".

\section{References}

[1] CHARLET, G., CORBEL, E., LAZARO, J., KLEKAMP, A., DISCHLER, R., TRAN, P., IDLER, W., MARDOYAN, H., KONCZYKOWSKA, A., JORGE, F., BIGO, S.: WDM Transmission at 6-Tbit/s Capacity Over Transatlantic Distance, Using 42.7-Gb/s Differential Phase-Shift Keying Without Pulse Carver, Journal of Lightwave Technology, 1/2005.

[2] YOSHIKANE, N., MORITA, I.: $1.14 \mathrm{~b} / \mathrm{s} / \mathrm{Hz}$ Spectrally Efficient 50 85.4-Gb/s Transmission Over $300 \mathrm{~km}$ Using Copolarized RZDQPSK Signals, Journal of Lightwave Technology, 1/2005, pp. 108-114.

[3] BIGO, S.: Optimizing terrestrial systems for $40 \mathrm{Gbit} / \mathrm{s}$ channel bit rates, Glasgow: ECOC 2005.

[4] GNAUCK, A. H., WINZER, P. J.: Optical PSK transmission, Journal of Lightwave Technology, 1/2005.

[5] AVLONITIS, N. S., YEATMAN, E. M.: Performance Evaluation of Optical DQPSK Using Saddle Point Approximation, Journal of Lightwave Technology, 3/2006

[6] SABOL, D.: WDM systems with DPSK keying, Diploma thesis, University of Zilina, 2006. 